\title{
$\frac{\{(8)\}}{\text { BIOENG }}$
}

\section{ARTMAP-FUZZY: SISTEMA INTELIGENTE APLICADO NA DETECÇÃO DE FALHAS DE UM TRATOR}

\author{
${ }^{1}$ Instituto Federal de São Paulo, Campus Salto, Salto, SP, Brasil \\ ${ }^{2}$ UNESP - Univ. Estadual Paulista, Faculdade de Engenharia de Ilha Solteira, Departamento \\ Matemática, Ilha Solteira, SP, Brasil
}

Article history: Received 16 September 2016; Received in revised form 24 October 2016; Accepted 25 October 2016; Available online 27 December 2016.

\section{RESUMO}

Este trabalho apresenta o desenvolvimento de um sistema inteligente que, por meio de uma rede neural artificial, atua na detecção de falhas estruturais em um trator. Simulou-se o trator por meio de um modelo numérico, construído através de equações diferenciais, o qual gera dados conforme se altera a velocidade e a distância entre as saliências no solo. Para a análise, identificação e caracterização dos dados simulados computacionalmente, foi codificado um sistema que utiliza conceitos da Teoria da Ressonância Adaptativa presentes na rede neural do tipo ARTMAP-Fuzzy. O principal objetivo deste sistema é inspecionar a estrutura do trator contribuindo para sua melhor conservação, indicando se ela se encontra em condições normais ou em situação de falha estrutural. Os resultados obtidos na aplicação da rede neural ao problema especificado mostraram-se ser eficientes e precisos.

Palavras-chave: redes neurais artificiais; ARTMAP-Fuzzy; detecção de falhas; trator

\section{ARTMAP-FUZZY: INTELLIGENT SYSTEM APPLIED TO FAULT DETECTION IN A TRACTOR}

\begin{abstract}
This paper presents the development of an intelligent system that, by an artificial neural network, works in the detection of structural flaws in a tractor. It was simulated a tractor by means of a numerical model built by differential equations, which generates data as to change the speed and the distance between the protrusions on the ground. For the analysis, identification and characterization of computationally simulated data was encoded a system that utilizes concepts of Adaptive Resonance Theory present in the ARTMAP-fuzzy neural network. The main objective of this system is to inspect the tractor structure contributing to its better conservation, indicating whether it is in normal conditions or structural failure situation. The results obtained in the application of neural network to the specified problem proved to be efficient and accurate.
\end{abstract}

Keywords: artificial neural network; ARTMAP-Fuzzy; failure detection; tractor

\footnotetext{
fabioch@mat.feis.unesp.br
} 


\section{INTRODUÇÃO}

A agricultura é uma das principais atividades econômicas da sociedade moderna e, como em todas as áreas, a informatização tem sido incorporada nos processos produtivos ao longo dos anos. A incorporação das tecnologias possibilitou a expansão da área cultivada e o aumento da produtividade.

Um crescimento ainda maior da produção agrícola depende, entre outros fatores, do nível de tecnologia em tratores usados na agricultura. Os tratores, para atenderem as exigências de custos e de produtividade, devem permitir a seus operadores a condição de exigir destas máquinas o máximo desempenho nas tarefas do campo. Sob certas condições do traçado e velocidade, o trator pode apresentar instabilidade e provocar vibrações que são prejudiciais à estrutura do trator ou, em casos mais extremos, o seu tombamento (KHOURY et al, 2004).

Desta forma é necessário desenvolver maneiras eficientes de monitorar a estrutura, visando detectar falhas em estados iniciais, intervir na sua propagação e, consequentemente, impedir o seu desgaste ou até mesmo o seu tombamento. Esta atual e importante linha de pesquisa é denominada Monitoramento da Integridade de Estruturas (SHM Structural Health Monitoring).

Conforme destacado por Hall (1999), um SHM deve satisfazer os seguintes requisitos: aquisição e processamento de dados; validação e análise de sinais; identificação e caracterização de falhas; interpretação de mudanças adversas em uma estrutura; auxiliar a tomada de decisões.

Toda estrutura passa por uma avaliação de testes de integridade

\section{MATERIAL E MÉTODO}

Primeiramente faz-se uma abordagem no conceito da rede neural ARTMAP-Fuzzy e seu algoritmo de treinamento. estrutural. Neste caso são realizadas avaliações não destrutivas (NDE Nondestructive Evaluation), visando estimar o grau de segurança e confiabilidade da estrutura.

Nos últimos anos, os sistemas de monitoramento de integridade estrutural vêm sofrendo uma evolução natural, devido ao avanço das tecnologias de sensores, materiais inteligentes, técnicas eficientes de processamento de dados e sinais, e principalmente a crescente demanda de se empregar tais ferramentas em tempo real.

Neste contexto, uma possível solução, para se desenvolver um SHM moderno e eficiente é a utilização de sensores inteligentes e/ou técnicas de computação inteligentes (redes neurais artificiais, lógica fuzzy, etc.), que viabilizam habilidades como a extração de conhecimentos e informações de processos complexos, facilitam a análise de sinais e proporcionam a automatização da tomada de decisões nos SHMs.

Neste trabalho, apresenta-se a proposta de uma metodologia para o desenvolvimento do SHM para solucionar o problema baseado em técnicas de computação inteligente (CI). Neste sentido, emprega-se uma rede neural artificial (RNA) ARTMAP-Fuzzy (CARPENTER et al., 1992) para realizar a análise e monitoramento da integridade do trator, devido as suas características de aprendizagem e reconhecimento de padrões, e por apresentarem bom desempenho em outros tipos de problemas de reconhecimento de padrões e diagnóstico, conforme destacado pelos autores em Lima et al. (2013) e TonelliNeto (2012).

\section{A rede neural ARTMAP-Fuzzy e seu algoritmo}

A rede neural artificial ARTMAPFuzzy possui uma arquitetura estável e 
plástica, que garante uma vantagem com relação a outros tipos de redes neurais artificiais. A rede ARTMAP-Fuzzy permite a inclusão de um módulo de treinamento continuado, o qual habilita a extração de conhecimento sem a necessidade de reiniciar o processo de treinamento quando novos padrões são apresentados, ao contrário do que acontece com a maioria das redes neurais (CARPENTER et al., 1992). A RNA ARTMAP-Fuzzy trata-se de um sistema de aprendizagem supervisionado composta de um par de módulos ART (CARPENTER e GROSSBERG, 1987), ARTa-Fuzzy e ARTb-Fuzzy, interconectados por um módulo de memória associativa inter-ART. Esta arquitetura de rede neural incorpora a teoria fuzzy, o operador lógico AND fuzzy $\left(^{\wedge}\right)$ habilitando o sistema neural de aprendizagem, em resposta aos padrões de entradas binários e analógicos, durante os intervalos [0 1] (CARPENTER et al., 1992).

Martins (2010) afirma que um mecanismo interno chamado matchtracking é responsável pelo processo auto regulatório da rede neural, no qual maximiza a generalização e minimiza o erro. Quando a rede neural faz uma predição errada, através de uma conexão associativa instruída, o parâmetro de monitoramento do módulo ARTa-Fuzzy é incrementado no mínimo necessário para corrigir o erro no módulo ARTa-Fuzzy. Segundo Lopes (2005), a figura 1 ilustra a arquitetura da rede neural ARTMAPFuzzy.

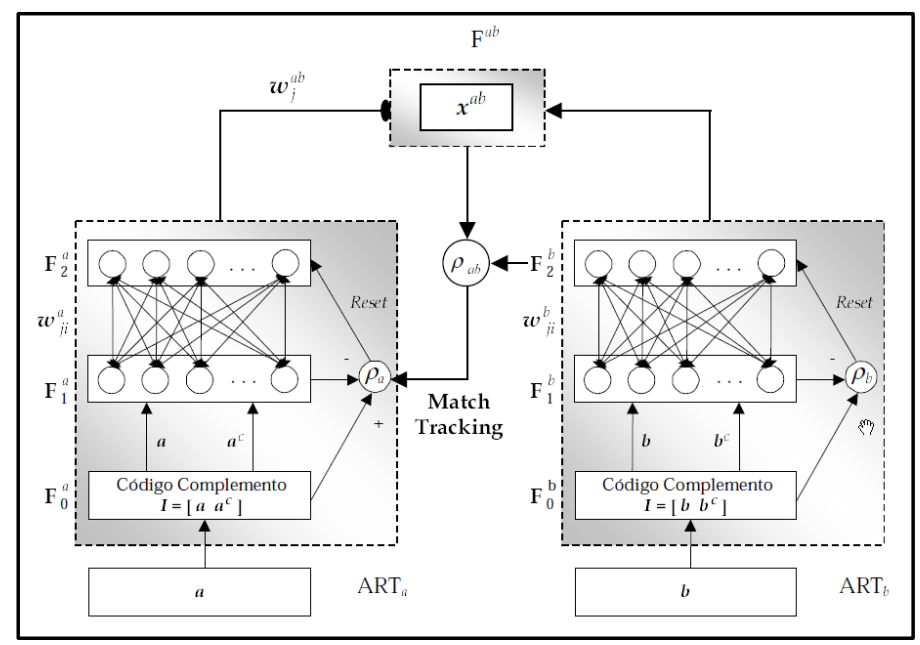

Figura 1. Arquitetura da Rede Neural Artificial ARTMAP-Fuzzy.

De acordo com Carpenter et al. (1992), a arquitetura ARTMAP-Fuzzy possui três parâmetros principais para $\mathrm{O}$ desenvolvimento, chamados:

- Parâmetro de escolha $\alpha(\alpha>0)$ : opera na categoria de seleção;

- Taxa de treinamento $\beta\left(\beta \in\left[\begin{array}{ll}0 & 1\end{array}\right]\right)$ : Controla a velocidade de adaptação da rede;

- Parâmetro de vigilância ( $\rho a, \rho b$ e $\rho a b \epsilon$ [0 1]): controla a ressonância da rede, nomeado os parâmetros responsáveis pelo número de categorias criadas.

Se $\rho$ possui um valor alto, a rede neural torna-se mais seletiva reduzindo sua capacidade de generalização. Se $\rho$ possui um valor pequeno, é reduzido o número de categorias criadas, maximizando a capacidade de generalização da rede ARTMAP-Fuzzy.

De acordo com Lopes (2005), a rede neural ARTMAP-Fuzzy consiste em dois módulos ART Fuzzy, o ARTa e o ARTb. E são apresentadas em seguida as etapas de como o algoritmo é executado durante a fase de treinamento.

Etapa 1: obtém-se os dados de entrada para o módulo ARTa e ARTb.

Etapa 2: efetua-se a normalização dos vetores A e B, respectivamente. Em (1) é 
possível observar a normalização do vetor de entrada do módulo ARTa.

$$
\bar{a}=\frac{a}{|a|}
$$

Etapa 3: inicialmente todos os pesos da rede neural possuem o valor igual a 1 , indicando que não existe categoria ativa.

$$
W_{j}^{a}=W_{k}^{b}=W_{j}^{a b}=1
$$

Etapa 4: faz-se a leitura dos parâmetros $\alpha, \beta, \rho_{a}, \rho_{b}, \rho_{a b}, \varepsilon$.

Etapa 5: efetua-se a codificação do complemento, preservando a amplitude da informação.

$$
\overline{a_{l}^{c}}=1-\overline{a_{l}}
$$

Etapa 6: então o vetor de entrada no campo $F_{0}$ será um vetor $2 \mathrm{M}$-dimensional.

$$
\begin{aligned}
& I=\left[\begin{array}{ll}
\bar{a} & \overline{a^{c}}
\end{array}\right] \\
& =\left[\begin{array}{llllll}
\overline{a_{1}} & \ldots & \bar{a}_{M} & \bar{a}_{1}^{c} & \ldots & \bar{a}_{M}^{c}
\end{array}\right]
\end{aligned}
$$

Etapa 7: obtém-se valores os quais poderão ser escolhidos como categoria.

$$
T_{j}=\frac{\left|I \wedge W_{j}\right|}{\alpha+\left|W_{j}\right|}
$$

Etapa 8: escolhe-se a categoria do módulo ARTb.

$$
T_{k}^{b}=\max \left\{T_{k}^{b}: k=1, \ldots, N_{b}\right\}
$$

Etapa 9: neste momento ocorre o teste de vigilância. Se houver ressonância, parte-se para a etapa 11, caso contrário, etapa 10.

$$
\frac{\left|I_{s} \wedge W_{k}^{b}\right|}{I_{s}} \geq \rho_{b}
$$

Etapa 10: reset. Após resetado volta-se para a etapa 8 .

$$
T_{k}^{b}=0
$$

Etapa 11: adapta-se os pesos do módulo ARTb.

$$
\begin{aligned}
W_{k}^{\text {novo }}=\beta\left(I_{s}\right. & \left.\wedge W_{k}^{\text {velho }}\right) \\
& +(1-\beta) W_{k}^{\text {velho }}
\end{aligned}
$$

Etapa 12: atualiza-se o vetor atividade $Y_{b}$.
Etapa 13: escolhe-se a categoria do módulo ARTa.

$$
T_{j}^{a}=\max \left\{T_{j}^{a}: j=1, \ldots, N_{a}\right\}
$$

Etapa 14: faz-se o teste de vigilância. Se houver ressonância, parte-se para a etapa 16, caso contrário, etapa 15.

$$
\frac{\left|I_{e} \wedge W_{j}^{a}\right|}{I_{e}} \geq \rho_{a}
$$

Etapa 15: reset. Após resetado volta-se para a etapa 13.

$$
T_{j}^{a}=0
$$

Etapa 16: realiza-se o match tracking. Se houver ressonância, parte-se para a etapa 19, caso contrário, etapa 17.

$$
\frac{\left|Y^{b} \wedge W_{j}^{a b}\right|}{Y^{b}} \geq \rho_{a b}
$$

Etapa 17: reset.

$$
T_{j}^{a}=0
$$

Etapa 18: acresce-se o parâmetro de vigilância, e volta-se para a etapa 13 .

$$
\rho_{a}=\frac{\left|I_{e} \wedge W_{j}^{a}\right|}{I_{e}}+\varepsilon
$$

Etapa 19: atualiza-se o vetor atividade de $\mathrm{F}_{2}$.

Etapa 20: adapta-se os pesos do módulo ARTa.

$$
\begin{aligned}
W_{j}^{\text {novo }}=\beta\left(I_{e}\right. & \left.\wedge W_{j}^{\text {velho }}\right) \\
& +(1-\beta) W_{j}^{\text {velho }}
\end{aligned}
$$

Etapa 21: adapta-se os pesos Inter-ART. Treinamento concluído.

\section{Desenvolvimento $\quad e \quad$ simulação computacional}

O principal objetivo deste artigo consiste no desenvolvimento de um sistema inteligente para o monitoramento de falhas estruturais em um trator. Os dados são obtidos através de simulações realizadas em um modelo numérico, construído através de equações 
diferenciais. O modelo do trator está ilustrado na figura 2 (SAKAI, 2001).

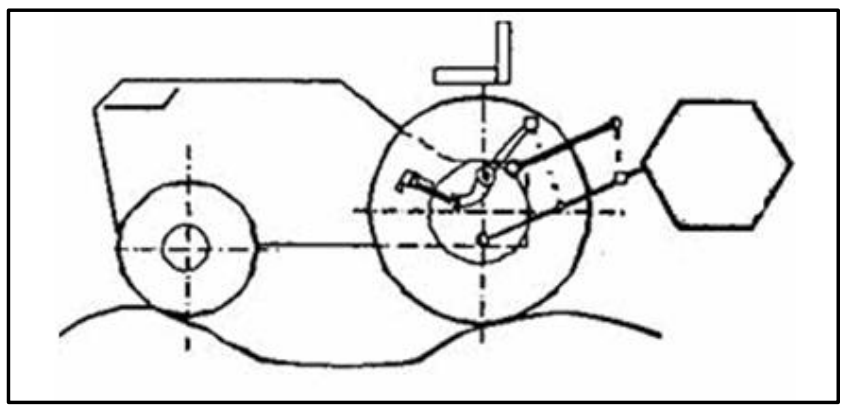

Figura 2. Modelo do trator.

Durante o trabalho no campo, as rodas traseiras e dianteiras de um trator podem perder o contato com o chão. Este fenômeno pode ocorrer de 3 modos.

- Somente as rodas dianteiras perdem contato com o chão (o trator empina a frente);

- Somente as rodas traseiras perdem contato com o chão (o trator empina a traseira);
- E as rodas traseiras e dianteiras perdem contato com o chão ao mesmo tempo (o trator dá um salto).

Diante disto, um modelo matemático simplificado em duas dimensões (horizontal e vertical) que descreve a dinâmica de um trator durante o salto é mostrado pela figura 3 .

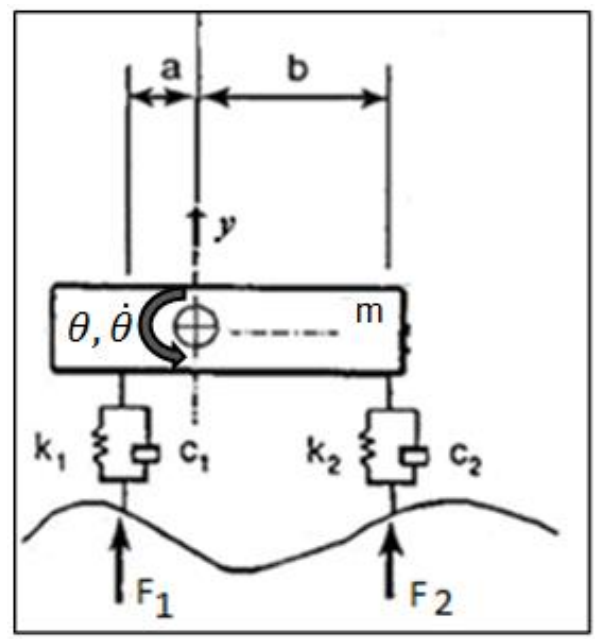

Figura 3. Modelo dinâmico.

A força de equilíbrio na direção vertical e do momento do salto é equacionada por:

$$
\begin{aligned}
& m \ddot{y}+F_{1}(y, \dot{y}, \theta, \dot{\theta})+F_{2}(y, \dot{\gamma}, \theta, \dot{\theta})+m g=0 \\
& J \ddot{\theta}+a F_{1}(y, \dot{y}, \theta, \dot{\theta})-b F_{(18)}(y, \dot{y}, \theta, \dot{\theta})=0
\end{aligned}
$$

Sendo:

$J=$ momento angular de inércia do centro de massa;

$F_{1}(\cdot)$ e $F_{2}(\cdot)=$ cargas dinâmicas nos pneus dianteiro e traseiro do trator, respectivamente, dadas por:

$$
\begin{aligned}
& F_{1}(y, \dot{y}, \theta, \dot{\theta})=k_{1}\left(y+a \theta-h_{1}(t)\right)+ \\
& c_{1}\left(\dot{y}+a \dot{\theta}-\dot{h}_{1}(t)\right) \\
& \quad F_{2}(y, \dot{y}, \theta, \dot{\theta})=k_{2}\left(y-b \theta-h_{2}(t)\right)+ \\
& \quad c_{2}\left(\dot{y}-b \dot{\theta}-\dot{h}_{2}(t)\right)
\end{aligned}
$$

Nas equações (19) e (20), temos respectivamente $h_{1}(t)$ e $h_{2}(t)$ as quais 
são excitações periódicas que simulam os desníveis da superfície: $h_{1}(t)=h_{0} \operatorname{sen}(\omega t)$ e $h_{2}(t)=h_{0} \operatorname{sen}(\omega t-\phi) \quad$ onde, $\quad \omega=\frac{2 \pi v}{L} \quad \mathrm{e}$ $\phi=\frac{2 \pi W_{B}}{L}$.

Observe que quando o trator:

- Empina a frente $F_{1}(\cdot)=0$. Logo: $k_{1}=c_{1}=0$;

- Empina a traseira $F_{2}(\cdot)=0$. Logo: $k_{2}=c_{2}=0$;

- Dá um salto $F_{1}(\cdot)=F_{2}(\cdot)=0$. Logo: $k_{1}=c_{1}=k_{2}=c_{2}=0$.

Para realizar as simulações das condições normais (base-line) e anormais (falha estrutural), no trator altera-se a distância entre as saliências consecutivas
$(\mathrm{L}=0,6 \mathrm{~m}, \quad \mathrm{~L}=0,4 \mathrm{~m}, \mathrm{~L}=0,3 \mathrm{~m}, \quad \mathrm{~L}=0,2 \mathrm{~m} \quad \mathrm{e}$ $\mathrm{L}=0,15 \mathrm{~m})$ e variam-se as velocidades $(\mathrm{v}=1 \mathrm{~m} / \mathrm{s}$ até $10 \mathrm{~m} / \mathrm{s})$ para cada $\mathrm{L}$. Sabe-se que $\mathrm{o}$ trator funciona $\mathrm{em}$ condições normais com $\mathrm{L}=0,6 \mathrm{~m}$ e a variação de $\mathrm{v}=1 \mathrm{~m} / \mathrm{s}$ a $3 \mathrm{~m} / \mathrm{s}$, e sendo as outras consideradas anormais, como $\mathrm{L}=0,15 \mathrm{~m}$ e velocidade $\mathrm{v}=6 \mathrm{~m} / \mathrm{s}$ que, sob certas condições do traçado e velocidade, o trator poderá apresentar instabilidade nas vibrações prejudicando o controle por parte do operador.

\section{Funcionamento do sistema}

$\mathrm{O}$ sistema inteligente de monitoramento e diagnóstico de falhas proposto neste artigo segue o fluxograma proposto por Lima et al. (2014) ilustrada na figura 4.

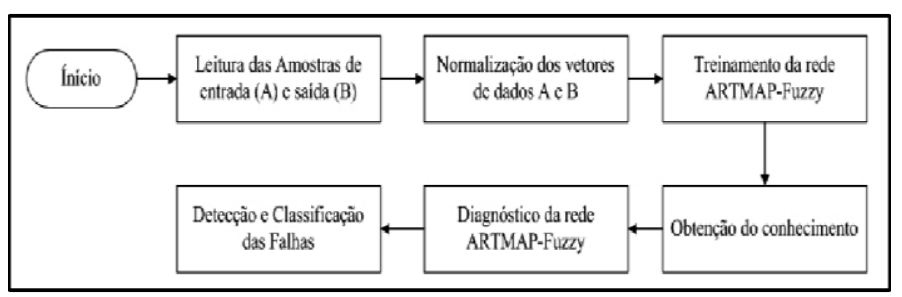

Figura 4. Funcionamento do sistema inteligente.

A fase de aquisição é um modelo numérico de um trator já mencionado anteriormente. $\mathrm{O}$ conjunto de entrada da rede ARTMAP-Fuzzy é composto por sinais de resposta em frequência.

A fase de treinamento da rede neural ARTMAP-Fuzzy é realizada de forma offline e é importante para ajustar os pesos e criar as categorias que serão utilizadas no diagnóstico dos dados. Nesta fase a rede é treinada com um conjunto de dados gerando uma quantidade de categorias igual ao número de sinais de entrada.

A fase de diagnóstico é realizada de forma online. Nesta fase um novo conjunto de sinais diferentes do conjunto de sinais utilizados na fase de treinamento deve ser apresentada a rede, e cada sinal será analisado individualmente. Todos os sinais são comparados com o conhecimento (categorias criadas na fase de treinamento) obtido pela rede neural ARTMAP-Fuzzy e assim, quando houver um casamento entre os padrões classifica-se em categoria o sinal apresentado a rede. Obtida a categoria correspondente a um determinado padrão é, portanto, identificado a intensidade (porcentagem de falha) e a localização da falha na estrutura. E para exemplificar os sinais analisados pelo sistema, pode-se observar um sinal normal através da figura 5 e um sinal anormal através da figura 6 . 


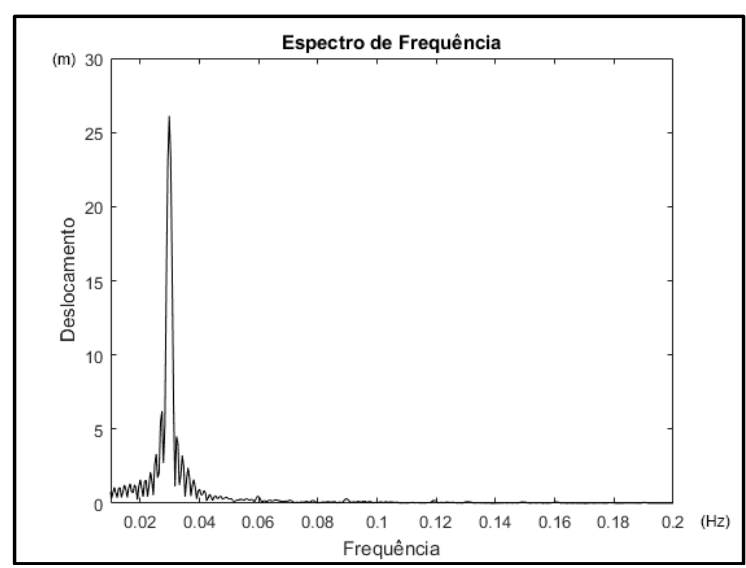

Figura 5. Representação de um sinal normal.

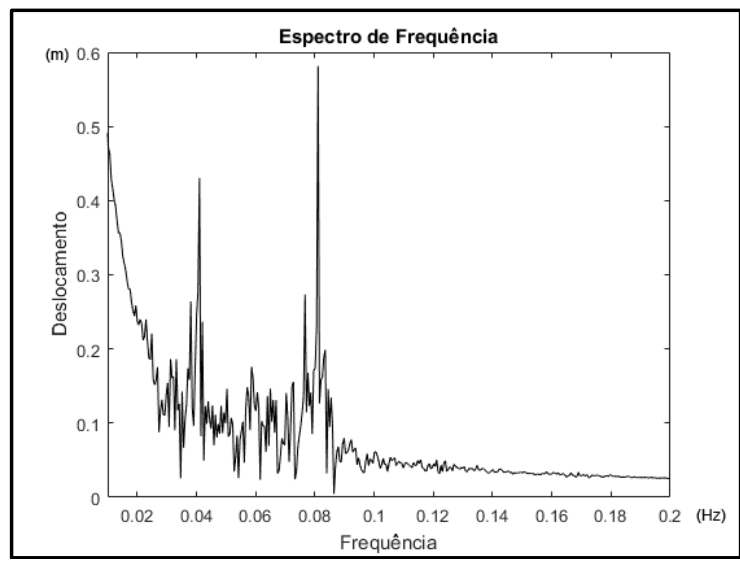

Figura 6. Representação de um sinal anormal.

\section{Cenários propostos para análise do} sistema

Para testar o sistema inteligente, foram propostos 5 cenários diferentes para treinamento e diagnóstico, representados a seguir por tabelas, onde L é a distância em metros entre as saliências, e estão dispostas em cinco linhas. Disposto em colunas estão as velocidades, de 1 a $10 \mathrm{~m} / \mathrm{s}$. Dentro das tabelas, a letra $\mathrm{T}$ significa que os dados foram utilizados para treinamento e a letra D para diagnóstico. Os cenários podem ser observados através das tabelas 1, 2, 3, 4 e 5. Sabe-se que são condições normais apenas os sinais onde $\mathrm{L}=0,6 \mathrm{~m}$ e velocidade igual a 1,2 ou $3 \mathrm{~m} / \mathrm{s}$. As demais situações, são todas de falha estrutural, ou seja, 47 situações.

Tabela 1. Cenário 1.

\begin{tabular}{c|c|c|c|c|c|c|c|c|c|c}
\hline $\mathbf{m} / \mathbf{s}$ & $\mathbf{1}$ & $\mathbf{2}$ & $\mathbf{3}$ & $\mathbf{4}$ & $\mathbf{5}$ & $\mathbf{6}$ & $\mathbf{7}$ & $\mathbf{8}$ & $\mathbf{9}$ & $\mathbf{1 0}$ \\
\hline $\mathrm{L}=0,6 \mathrm{~m}$ & $\mathrm{~T}$ & $\mathrm{~T}$ & $\mathrm{~T}$ & $\mathrm{~T}$ & $\mathrm{~T}$ & $\mathrm{~T}$ & $\mathrm{~T}$ & $\mathrm{D}$ & $\mathrm{D}$ & $\mathrm{D}$ \\
\hline $\mathrm{L}=0,4 \mathrm{~m}$ & $\mathrm{~T}$ & $\mathrm{~T}$ & $\mathrm{~T}$ & $\mathrm{~T}$ & $\mathrm{~T}$ & $\mathrm{~T}$ & $\mathrm{~T}$ & $\mathrm{D}$ & $\mathrm{D}$ & $\mathrm{D}$ \\
\hline $\mathrm{L}=0,3 \mathrm{~m}$ & $\mathrm{~T}$ & $\mathrm{~T}$ & $\mathrm{~T}$ & $\mathrm{~T}$ & $\mathrm{~T}$ & $\mathrm{~T}$ & $\mathrm{~T}$ & $\mathrm{D}$ & $\mathrm{D}$ & $\mathrm{D}$ \\
\hline $\mathrm{L}=0,2 \mathrm{~m}$ & $\mathrm{~T}$ & $\mathrm{~T}$ & $\mathrm{~T}$ & $\mathrm{~T}$ & $\mathrm{~T}$ & $\mathrm{~T}$ & $\mathrm{~T}$ & $\mathrm{D}$ & $\mathrm{D}$ & $\mathrm{D}$ \\
\hline $\mathrm{L}=0,15 \mathrm{~m}$ & $\mathrm{~T}$ & $\mathrm{~T}$ & $\mathrm{~T}$ & $\mathrm{~T}$ & $\mathrm{~T}$ & $\mathrm{~T}$ & $\mathrm{~T}$ & $\mathrm{D}$ & $\mathrm{D}$ & $\mathrm{D}$ \\
\hline
\end{tabular}


Tabela 2. Cenário 2.

\begin{tabular}{c|c|c|c|c|c|c|c|c|c|c}
\hline $\mathbf{m} / \mathbf{s}$ & $\mathbf{1}$ & $\mathbf{2}$ & $\mathbf{3}$ & $\mathbf{4}$ & $\mathbf{5}$ & $\mathbf{6}$ & $\mathbf{7}$ & $\mathbf{8}$ & $\mathbf{9}$ & $\mathbf{1 0}$ \\
\hline $\mathrm{L}=0,6 \mathrm{~m}$ & $\mathrm{~T}$ & $\mathrm{~T}$ & $\mathrm{~T}$ & $\mathrm{~T}$ & $\mathrm{~T}$ & $\mathrm{D}$ & $\mathrm{T}$ & $\mathrm{D}$ & $\mathrm{T}$ & $\mathrm{D}$ \\
\hline $\mathrm{L}=0,4 \mathrm{~m}$ & $\mathrm{~T}$ & $\mathrm{~T}$ & $\mathrm{~T}$ & $\mathrm{~T}$ & $\mathrm{~T}$ & $\mathrm{D}$ & $\mathrm{T}$ & $\mathrm{D}$ & $\mathrm{T}$ & $\mathrm{D}$ \\
\hline $\mathrm{L}=0,3 \mathrm{~m}$ & $\mathrm{~T}$ & $\mathrm{~T}$ & $\mathrm{~T}$ & $\mathrm{~T}$ & $\mathrm{~T}$ & $\mathrm{D}$ & $\mathrm{T}$ & $\mathrm{D}$ & $\mathrm{T}$ & $\mathrm{D}$ \\
\hline $\mathrm{L}=0,2 \mathrm{~m}$ & $\mathrm{~T}$ & $\mathrm{~T}$ & $\mathrm{~T}$ & $\mathrm{~T}$ & $\mathrm{~T}$ & $\mathrm{D}$ & $\mathrm{T}$ & $\mathrm{D}$ & $\mathrm{T}$ & $\mathrm{D}$ \\
\hline $\mathrm{L}=0,15 \mathrm{~m}$ & $\mathrm{~T}$ & $\mathrm{~T}$ & $\mathrm{~T}$ & $\mathrm{~T}$ & $\mathrm{~T}$ & $\mathrm{D}$ & $\mathrm{T}$ & $\mathrm{D}$ & $\mathrm{T}$ & $\mathrm{D}$ \\
\hline
\end{tabular}

Tabela 3. Cenário 3.

\begin{tabular}{c|c|c|c|c|c|c|c|c|c|c}
\hline $\mathbf{m} / \mathbf{s}$ & $\mathbf{1}$ & $\mathbf{2}$ & $\mathbf{3}$ & $\mathbf{4}$ & $\mathbf{5}$ & $\mathbf{6}$ & $\mathbf{7}$ & $\mathbf{8}$ & $\mathbf{9}$ & $\mathbf{1 0}$ \\
\hline $\mathrm{L}=0,6 \mathrm{~m}$ & $\mathrm{~T}$ & $\mathrm{~T}$ & $\mathrm{~T}$ & $\mathrm{~T}$ & $\mathrm{D}$ & $\mathrm{T}$ & $\mathrm{T}$ & $\mathrm{T}$ & $\mathrm{T}$ & $\mathrm{T}$ \\
\hline $\mathrm{L}=0,4 \mathrm{~m}$ & $\mathrm{D}$ & $\mathrm{D}$ & $\mathrm{D}$ & $\mathrm{D}$ & $\mathrm{T}$ & $\mathrm{T}$ & $\mathrm{T}$ & $\mathrm{T}$ & $\mathrm{T}$ & $\mathrm{T}$ \\
\hline $\mathrm{L}=0,3 \mathrm{~m}$ & $\mathrm{~T}$ & $\mathrm{~T}$ & $\mathrm{D}$ & $\mathrm{D}$ & $\mathrm{D}$ & $\mathrm{D}$ & $\mathrm{T}$ & $\mathrm{T}$ & $\mathrm{T}$ & $\mathrm{T}$ \\
\hline $\mathrm{L}=0,2 \mathrm{~m}$ & $\mathrm{~T}$ & $\mathrm{D}$ & $\mathrm{T}$ & $\mathrm{T}$ & $\mathrm{T}$ & $\mathrm{T}$ & $\mathrm{D}$ & $\mathrm{D}$ & $\mathrm{D}$ & $\mathrm{T}$ \\
\hline $\mathrm{L}=0,15 \mathrm{~m}$ & $\mathrm{D}$ & $\mathrm{T}$ & $\mathrm{T}$ & $\mathrm{T}$ & $\mathrm{T}$ & $\mathrm{T}$ & $\mathrm{T}$ & $\mathrm{T}$ & $\mathrm{T}$ & $\mathrm{D}$ \\
\hline
\end{tabular}

Tabela 4. Cenário 4.

\begin{tabular}{c|c|c|c|c|c|c|c|c|c|c}
\hline $\mathbf{m} / \mathbf{s}$ & $\mathbf{1}$ & $\mathbf{2}$ & $\mathbf{3}$ & $\mathbf{4}$ & $\mathbf{5}$ & $\mathbf{6}$ & $\mathbf{7}$ & $\mathbf{8}$ & $\mathbf{9}$ & $\mathbf{1 0}$ \\
\hline $\mathrm{L}=0,6 \mathrm{~m}$ & $\mathrm{~T}$ & $\mathrm{~T}$ & $\mathrm{~T}$ & $\mathrm{~T}$ & $\mathrm{~T}$ & $\mathrm{~T}$ & $\mathrm{~T}$ & $\mathrm{~T}$ & $\mathrm{~T}$ & $\mathrm{~T}$ \\
\hline $\mathrm{L}=0,4 \mathrm{~m}$ & $\mathrm{D}$ & $\mathrm{T}$ & $\mathrm{D}$ & $\mathrm{T}$ & $\mathrm{D}$ & $\mathrm{T}$ & $\mathrm{D}$ & $\mathrm{T}$ & $\mathrm{D}$ & $\mathrm{T}$ \\
\hline $\mathrm{L}=0,3 \mathrm{~m}$ & $\mathrm{~T}$ & $\mathrm{D}$ & $\mathrm{T}$ & $\mathrm{D}$ & $\mathrm{T}$ & $\mathrm{D}$ & $\mathrm{T}$ & $\mathrm{D}$ & $\mathrm{T}$ & $\mathrm{D}$ \\
\hline $\mathrm{L}=0,2 \mathrm{~m}$ & $\mathrm{D}$ & $\mathrm{T}$ & $\mathrm{D}$ & $\mathrm{T}$ & $\mathrm{D}$ & $\mathrm{T}$ & $\mathrm{D}$ & $\mathrm{T}$ & $\mathrm{D}$ & $\mathrm{T}$ \\
\hline $\mathrm{L}=0,15 \mathrm{~m}$ & $\mathrm{~T}$ & $\mathrm{~T}$ & $\mathrm{~T}$ & $\mathrm{~T}$ & $\mathrm{~T}$ & $\mathrm{~T}$ & $\mathrm{~T}$ & $\mathrm{~T}$ & $\mathrm{~T}$ & $\mathrm{~T}$ \\
\hline
\end{tabular}

Tabela 5. Cenário 5.

\begin{tabular}{c|c|c|c|c|c|c|c|c|c|c}
\hline $\mathbf{m} / \mathbf{s}$ & $\mathbf{1}$ & $\mathbf{2}$ & $\mathbf{3}$ & $\mathbf{4}$ & $\mathbf{5}$ & $\mathbf{6}$ & $\mathbf{7}$ & $\mathbf{8}$ & $\mathbf{9}$ & $\mathbf{1 0}$ \\
\hline $\mathrm{L}=0,6 \mathrm{~m}$ & $\mathrm{~T}$ & $\mathrm{D}$ & $\mathrm{T}$ & $\mathrm{D}$ & $\mathrm{T}$ & $\mathrm{D}$ & $\mathrm{T}$ & $\mathrm{D}$ & $\mathrm{T}$ & $\mathrm{D}$ \\
\hline $\mathrm{L}=0,4 \mathrm{~m}$ & $\mathrm{~T}$ & $\mathrm{~T}$ & $\mathrm{~T}$ & $\mathrm{~T}$ & $\mathrm{~T}$ & $\mathrm{~T}$ & $\mathrm{~T}$ & $\mathrm{~T}$ & $\mathrm{~T}$ & $\mathrm{~T}$ \\
\hline $\mathrm{L}=0,3 \mathrm{~m}$ & $\mathrm{D}$ & $\mathrm{T}$ & $\mathrm{D}$ & $\mathrm{T}$ & $\mathrm{D}$ & $\mathrm{T}$ & $\mathrm{D}$ & $\mathrm{T}$ & $\mathrm{D}$ & $\mathrm{T}$ \\
\hline $\mathrm{L}=0,2 \mathrm{~m}$ & $\mathrm{~T}$ & $\mathrm{~T}$ & $\mathrm{~T}$ & $\mathrm{~T}$ & $\mathrm{~T}$ & $\mathrm{~T}$ & $\mathrm{~T}$ & $\mathrm{~T}$ & $\mathrm{~T}$ & $\mathrm{~T}$ \\
\hline $\mathrm{L}=0,15 \mathrm{~m}$ & $\mathrm{~T}$ & $\mathrm{D}$ & $\mathrm{T}$ & $\mathrm{D}$ & $\mathrm{T}$ & $\mathrm{D}$ & $\mathrm{T}$ & $\mathrm{D}$ & $\mathrm{T}$ & $\mathrm{D}$ \\
\hline
\end{tabular}

Após diversas execuções, a melhor configuração dos parâmetros independente do cenário aplicado, pode ser observada na tabela 6 .

Tabela 6. Valores dos parâmetros utilizados pela rede ARTMAP-Fuzzy.

\begin{tabular}{l|l}
\hline Parâmetros & Valores \\
\hline $\boldsymbol{\alpha}$ & 0,1 \\
\hline $\boldsymbol{\beta}$ & 1 \\
\hline $\boldsymbol{\rho}_{\mathbf{a}}$ & 0,45 \\
\hline $\boldsymbol{\rho}_{\mathbf{b}}$ & 1 \\
\hline $\boldsymbol{\rho}_{\mathbf{a b}}$ & 1 \\
\hline $\boldsymbol{\xi}$ & 0,0001 \\
\hline
\end{tabular}

É importante destacar que foi possível estabelecer um valor para $\rho_{a}$ relativamente baixo, devido os sinais apresentarem grande diferença entre condição normal e com falha, conforme visto anteriormente através da figura 6 . 
O algoritmo foi desenvolvido em MATLAB ${ }^{\circledR}$, utilizando-se a versão 6.5 , e os testes realizados em um PC Intel Core 2

\section{RESULTADOS E DISCUSSÃO}

Para o treinamento da rede neural foi utilizado um conjunto de dados com 35 sinais. A quantidade de sinais utilizadas para o treinamento, representa $70 \%$ dos dados disponíveis. Com objetivo de avaliar a rede neural na fase de diagnóstico foi usado um conjunto de dados com 15 sinais. Os dados utilizados na fase de diagnóstico
Duo $2.4 \mathrm{GHz}$, 6GB de memória RAM, e sistema operacional Windows 8 Professional 64 bits,

representam $30 \%$ dos dados disponíveis. A base de dados simulada apresenta 47 padrões distintos de falhas e apenas $3 \mathrm{em}$ condições normais.

$\mathrm{Na}$ tabela 7 apresentam-se os resultados obtidos pelo sistema SHM aplicado aos dados do trator. O resultado é o mesmo para os cinco cenários propostos.

Tabela 7. Resultados obtidos aplicando rede ARTMAP-Fuzzy.

\begin{tabular}{l|c|c}
\cline { 2 - 3 } & Fase de Treinamento & Fase de Diagnóstico \\
\hline $\begin{array}{l}\text { Amostras } \\
\text { Utilizadas }\end{array}$ & 35 & 15 \\
\hline $\begin{array}{l}\text { Classificações } \\
\text { Corretas }\end{array}$ & 35 & 15 \\
\hline $\begin{array}{l}\text { Classificações } \\
\text { Erradas }\end{array}$ & 0 & 0 \\
\hline Acerto $(\%)$ & 100 & 100 \\
\hline Tempo $(m s)$ & 300 & 90 \\
\hline
\end{tabular}

Os resultados apresentados na tabela 7 foram obtidos a partir da melhor configuração do sistema, sendo possível somente após uma fase experimental de

\section{CONCLUSÕES}

O sistema de diagnóstico de falhas em estruturas proposto neste estudo apresentou ótimos resultados, alcançando um índice de acerto de $100 \%$ quando executado com a sua melhor configuração. A rede neural ARTMAP-Fuzzy mostrou-se apta para ser utilizada como um sistema de tempo real, pois a fase de treinamento que é mais demorada, pode ser realizada de forma off-line, enquanto que a fase de diagnóstico obteve um tempo de resposta de 6 milissegundos por sinal analisado, tempo este aceitável para um sistema de tomada de decisão online. Portanto, testes e ajustes de parâmetros. O índice de acerto igual a $100 \%$ demonstra que o sistema SHM proposto apresenta um ótimo desempenho. conclui-se que o sistema para monitoramento de falhas em estruturas proposto a partir de uma rede neural artificial ARTMAP-Fuzzy é confiável e preciso quando utilizado com objetivo de manter a integridade de estruturas mecânicas.

Um dos motivos da resistência a tração da fibra de bambu ser maior que a da cana pode ser entendido utilizando os conhecimentos de Rudall (2007), pois através dos resultados da microtomografia da fibra de bambu, nota-se que a quantidade de xilema, que tem função 
estrutural, é maior que a da fibra da cana, e que, assim também, a concentração de

\section{REFERÊNCIAS BIBLIOGRÁFICAS}

CARPENTER, G. A. et al. Fuzzy ARTMAP: a neural network architecture for incremental supervised learning of analog multidimensional maps. IEEE Transactions on Neural Networks, v. 3, n. 5, p. 698-713, set 1992.

CARPENTER, G. A., GROSSBERG, S., A massively parallel architecture for a selforganizing neural pattern recognition machine. Computer Vision, Graphics, and Image Processing, v. 37, n. 1, p. 54-115, 1987.

HALL, S. R., The effective management and use of structural health data. International Workshop on Structural Health Monitoring, p. 265-275, 1999.

KHOURY, J. K. J., et al. Modelagem da estabilidade de tratores agrícolas de pneus. Pesq. Agropec. Bras., Brasília, v. 39, n. 5, p. 459-468, mai. 2004.

LIMA, F. P. A., et al. ARTMAP-Fuzzy artificial neural network applied to the monitoring and fault identification in structural. Lecture Notes in Information Technology, Korea, v. 39, p. 3-9, 2013.

LIMA, F. P. A. et al., Diagnóstico de falhas em estruturas mecânicas utilizando rede neural artificial ARTMAP-Fuzzy. Congresso de Matemática Aplicada e Computacional - CMAC, Curitiba, 1, 2014. 1-6. lignina nesta região possa ser maior que em outras áreas da planta.

LOPES, M. L. M., Desenvolvimento de redes neurais para previsão de cargas elétricas de sistemas de energia elétrica, 2005. 149. Tese (Doutorado em Engenharia Elétrica) - Faculdade de Engenharia Elétrica, Universidade Estadual Paulista - UNESP, Ilha Solteira, 2005.

MARTINS, J. R. D., Detecção e classificação de curtos-circuitos em sistemas de distribuição usando rede neural artificial ARTMAP nebulosa, 2010. $84 \mathrm{f}$. Dissertação (Mestrado em Engenharia Elétrica) - Faculdade de Engenharia Elétrica, Universidade Estadual Paulista UNESP, Ilha Solteira, 2010.

MATLAB (R2013a). $8.1 \quad$ Version, Mathworks Company

SAKAI, K., Nonlinear dynamics and chaos in agricultural systems. $1^{\text {a }}$. ed. Amsterdam: Elsevier Science B.V., 2001.

TONELLI-NETO, M. S., Formulação do controle preventivo em sistemas de distribuição de energia elétrica baseada na lógica fuzzy e redes neurais. 2012. 137. Dissertação (Mestrado em Engenharia Elétrica) - Faculdade de Engenharia Elétrica, Universidade Estadual Paulista UNESP, Ilha Solteira, 2012. 